\title{
Implementación y análisis del método de aula invertida: un estudio de caso en Bachillerato
}

\section{Implementation and analysis of a flipped classroom experience: a case study in upper secondary school}

\author{
Guillermo Herrera Sierra ${ }^{1}$, María Paz Prendes Espinosa ${ }^{2}$ \\ ${ }^{1}$ Consejería de Educación de la Junta de Andalucía, España (gherrera.economia@ gmail.com) \\ ${ }^{2}$ Departamento de Didáctica y Organización Escolar, Universidad de Murcia, España (pazprend@um.es)
}

\section{RESUMEN:}

Las necesidades de la sociedad del conocimiento nos llevan a que los estudiantes deban ser formados bajo perspectivas y planteamientos diferentes a como han sido formados los de generaciones anteriores. Los docentes se han enfrentado a la necesidad de realizar un replanteamiento metodológico fomentando la incorporación en las aulas de metodologías activas. Entre estas alternativas metodológicas se encuentra el aula invertida (flipped classroom) que modifica el orden metodológico tradicional, se traslada fuera del aula la exposición de contenidos y el tiempo en el aula se dedica a las tareas prácticas.

En este artículo se presentan los resultados de una experiencia de innovación educativa basada en la metodología del aula invertida en la materia de Economía con alumnado de primero de Bachillerato. Se diseñó e implementó la unidad didáctica "El dinero y la política monetaria" y se recogieron las opiniones del alumnado a través de un cuestionario que, junto con la observación del docente en el aula, muestran una opinión positiva del alumnado acerca de la metodología, especialmente en cuanto a la atención que reciben del profesor frente a la de las clases impartidas con metodologías tradicionales.

PALABRAS CLAVE: TIC, AULA INVERTIDA, EDUCACIÓN ECONÓMICA, ENSEÑANZA SECUNDARIA.

ABSTRACT:
In current information society students should be educated under different learning strategies from those used with previous generations. Educators are reconsidering methodological strategies fostering active learning strategies instead of direct instruction. Among active learning strategies we find Flipped Classroom, which switchs traditional learning order. Direct instruction is transferred out to the class and in class time is dedicated to tasks, discussions or problems solving.

On this article, we show the results of the application of Flipped Classroom in a teaching unit of economics in upper secondary school. After its design and implementation of the teaching unit "Money and monetary policy", we applied a questionnaire and we used teacher's observation during the process. After the analysis of collected data, our main conclusion is that students show a positive opinion, specially about the improvements in the attention paid by teachers in the class in comparison with the traditional classes.

$\begin{array}{lcc}\text { KEYWORDS: } & \text { ICT, FLIPPED } & \text { CLASSROOM, } \\ \text { ECONOMICS } & \text { EDUCATION, } & \text { SECONDARY } \\ \text { EDUCATION } & & \end{array}$




\section{INTRODUCCIÓN}

El modelo de educación que ha prevalecido está anclado en la época en la que fue diseñado, la revolución industrial (Bergman y Sams, 2012), se basa excesivamente en la enseñanza y no en el aprendizaje, es un modelo de reproducción en el que las singularidades o las diferencias personales tienen poca cabida (Tourón y Santiago, 2015). El profesor que es el que sabe, habla durante la clase mientras que los estudiantes, que no saben, se dedican a escuchar y tomar notas. Los resultados de la investigación educativa nos han permitido saber que el conocimiento no se puede transmitir de manera verbal y que, para enseñar, se deben facilitar las condiciones necesarias para que los estudiantes aprendan por sí mismos, bien mediante el descubrimiento o bien ayudándoles a pensar.

En consecuencia, los docentes se han enfrentado a la necesidad de realizar un replanteamiento metodológico en cuanto a la forma de impartir sus materias (Robledo et al., 2015), especialmente las metodologías activas que implican al alumnado han demostrado mejorar sus resultados (Norman y Wills, 2015), comprenden un amplio abanico de estrategias de enseñanza aprendizaje diferentes (Carr, Palmer, y Hagel, 2015) y se fundamentan en que el alumno guiado por el profesor asuma una mayor responsabilidad y autonomía en su proceso de aprendizaje.

La época en la que vivimos está marcada por fuertes cambios, quizá más tecnológicos que pedagógicos, que afectan a la educación, la escuela, y a los modelos tecnológicos que en ella empleamos (Tourón y Santiago, 2015). La introducción de las tecnologías de la información y comunicación (TIC) ha provocado cambios en todos los hábitos de la sociedad actual, en particular en la educación donde ha derivado en la aparición de múltiples y variadas propuestas dirigidas a la mejora (Merla y Yáñez, 2016). En este escenario, la enseñanza semipresencial (blended learning) se configura como una alternativa posible para una formación que trascienda los espacios del aula y se traslade a todas las esferas de la vida, propuestas que buscan combinar lo mejor de la formación presencial con las funcionalidades de la formación en línea.

En el ámbito de la educación semipresencial podría situarse la metodología del aula invertida (Prieto, Prieto y Del Pino, 2016), la cual promueve la presentación de contenidos fuera del aula pero, a diferencia de los planteamientos generales de la enseñanza semipresencial, en el aula invertida no se contempla una reducción de los tiempos dedicados a situaciones cara a cara, sino un uso diferente de estos tiempos (Llamazares, 2015).

El aula invertida es un modelo relativamente nuevo en educación que se centra en el aprendizaje basado en los alumnos (Sohrabi y Iraj, 2016). La idea de aula invertida se basa en modificar el orden metodológico tradicional, sustituyendo el trabajo del aula por el de casa y a la inversa (García-Barrera, 2013). Se traslada fuera del aula la exposición de contenidos y, por tanto, el trabajo a niveles cognitivos más bajos (Marqués, 2016), mientras que en el aula el docente actúa de manera más personalizada, orientando a los estudiantes en lugar de impartiendo clases magistrales. Las tecnologías, por tanto, sirven aquí de apoyo para sustituir las clases magistrales y dedicar el tiempo presencial de clase a una enseñanza activa. Foldnes (2016) define el aula invertida como un tipo de enseñanza semipresencial donde las clases tradicionales son trasladadas fuera del aula a través de vídeos online, liberando tiempo en el aula de manera que el docente puede utilizar metodologías más activas que las utilizadas tradicionalmente.

Merla y Yáñez (2016) establecen las siguientes diferencias entre el aula invertida y el aula tradicional.

En el aula tradicional:

- El docente presenta contenidos a través de clases magistrales y asigna actividades o tareas para ser realizadas en casa.

- Los estudiantes reciben los contenidos en el aula y completan tareas o actividades en casa.

En el aula invertida:

- El docente guía a los estudiantes, dispone de tiempo en el aula para interactuar con cada estudiante y atenderlo de acuerdo a sus necesidades personales.

- Los estudiantes son responsables de su propio aprendizaje, estudian a su propio ritmo y estilo contenidos que se encuentran disponibles en línea a través de diferentes modelos y formatos para su distribución.

- Las tareas, actividades o proyectos son realizadas en clase.

- Los estudiantes pueden detener y volver a ver el material tantas veces como sea necesario.

Aunque el concepto es joven, la metodología del aula invertida está ganando popularidad en contextos educativos y prensa especializada (Tucker, 2012). El desarrollo de nuevas tecnologías asíncronas de comunicación ha favorecido la introducción del aula 
invertida en estos últimos años (Ranalli y Moore, 2016). Diferentes estudios realizados en España y en distintos niveles educativos demuestran cómo la metodología del aula invertida incide positivamente en el alumnado: en Educación Primaria (Nuñez y Gutiérrez, 2016, Parra y Gutiérrez 2017); en Educación Secundaria (Barao y Palau, 2016; Calvillo, 2014; Fornons y Palau, 2016; Gómez, Castro, y Toledo, 2015, Salmerón, 2015); en Formación Profesional (Fernández-Gámez y GuerraMartín, 2016) y en enseñanzas universitarias (Barreras, 2016; Prieto et al., 2016; Rodríguez, Fernández, y Vega, 2015; Sáez y Ros, 2014; Velilla, Cazanave, Cañizares, y Fernández, 2014; Sosa y Palau, 2018; Salas y Lugo, 2019).

Sin embargo, todo apunta a que las tecnologías, o quizás el uso que se hace de las tecnologías en el aula, influyen de manera diversa en función de la asignatura (Harrison et al., 2003; Rodríguez, Nieto, y Sumoza, 2016). En este sentido, añadir que no se tiene constancia de la aplicación de la metodología del aula invertida en la materia de Economía de primero de Bachillerato con anterioridad a nuestra experiencia.

\section{MATERIAL Y MÉTODO}

\subsection{Objetivos del estudio}

Esta experiencia de innovación educativa pretende promover la utilización del método de aula invertida en la asignatura de Economía de Primero de Bachillerato. Pretendemos conocer la opinión del alumnado sobre la experiencia y, con ello, analizar su viabilidad para el futuro.

Partiendo de este objetivo general se extraen los siguientes objetivos específicos:

- Diseñar, implementar y analizar críticamente una experiencia basada en el aula invertida en la unidad didáctica "El dinero y la política monetaria".

- Analizar las opiniones del alumnado acerca de la metodología

- Analizar si la metodología del aula invertida mejora la atención personalizada y la atención a la diversidad.

\subsection{Contexto y participantes}

La experiencia de innovación educativa se llevó a cabo en un instituto de Educación Secundaria de Andalucía de titularidad pública. El centro se encuentra en una localidad costera en una zona de nivel socioeconómico medio.
El grupo de primero de Bachillerato con el que se ha desarrollado esta experiencia está compuesto por 22 sujetos (10 alumnos y 12 alumnas) de entre 16 y 17 años de edad. Todos cuentan con conexión a Internet en sus domicilios y dispositivos de uso personal para ver vídeos en línea.

\subsection{Método}

Este estudio se aborda desde la perspectiva de la investigación evaluativa, que tiene por objeto valorar la eficacia o éxito de una acción educativa (Cabrera, 1987) y un diseño de estudio de caso enfocado desde los principios de la investigación-acción en el marco de las metodologías orientadas a la mejora de la práctica educativa. Podemos afirmar que el propósito fundamental de la investigación evaluativa es recoger información pertinente y válida para emitir juicios de valor orientados a la toma de decisiones para la mejora y el cambio. Para Pérez Serrano et al. (2007) la evaluación puede tener entre sus fines tomar decisiones sobre continuar o no continuar con una práctica educativa o mejorar la práctica a través de la compresión de la misma.

La experiencia a evaluar como caso de estudio se sustenta en una innovación educativa basada en la implementación de metodologías de aula invertida y sus correspondientes recursos digitales -diseñados y producidos ad hoc para la experiencia-. La finalidad última es conocer en profundidad qué ha ocurrido en el aula y poder tomar decisiones de mejora de cara a futuras experiencias en esta misma línea de trabajo.

\subsection{Técnicas e instrumentos de recogida de información}

Dadas las características de esta investigación, se han considerado los siguientes instrumentos de recogida de información:

- La observación participante mediante registro diario por parte del profesor.

- Análisis de las producciones de los alumnos.

- Cuestionario final -adaptado de Calvillo (2014) y Núñez y Gutiérrez (2016)-.

Las hojas de registro para la observación y para el análisis de las producciones de los alumnos se diseñaron específicamente para esta investigación en relación a los objetivos de evaluación y se validaron por juicio de expertos.

Además de recoger esta información de modo sistemático, se llevó a cabo una sesión de debate en grupo informal para comentar y valorar los resultados, recogiendo así información cualitativa de gran interés con la cual poder analizar de modo global la experiencia al concluir la misma. 


\section{DISEÑO DE LA UNIDAD DIDÁCTICA}

\subsection{Herramientas utilizadas}

Esta experiencia de innovación educativa se ha desarrollado en la unidad didáctica "El dinero y la política monetaria" relativos al bloque $\mathrm{V}$ de contenidos para la materia de Economía del Real Decreto 1105/2014 por el que se establece el currículo básico de la Educación Secundaria Obligatoria y el Bachillerato.

Para la puesta en marcha de esta experiencia se consideraron necesarias diferentes herramientas:

- Edmodo: como herramienta de comunicación fuera del aula y entrega de trabajos y tareas en formato digital. Es una de las herramientas de microblogging que se utiliza en educación para organizar contenidos $\mathrm{o}$ asignaciones con una comunicación e interacción constante entre profesores y alumnos (Sáez, Fernández, y García, 2012).

- Edpuzzle: facilita el visionado de vídeos interactivos. Es una herramienta favorecedora del aprendizaje activo y autoaprendizaje (Cakir, 2016; Jancsó, 2017; Palazón-herrera, 2016; Prieto Calvo et al., 2016); nos permite personalizar vídeos preparados por otros y transformarlos para usos educativos (Jancsó, 2017). Como señalan Hall y Dufrene (2016), el aula invertida no debe limitarse a seleccionar vídeos que los alumnos vean en sus casas, sino que es recomendable contemplar actividades que motiven a los participantes en la realización del trabajo fuera del aula, como tests o preguntas de intervención a lo largo de los vídeos que enriquezcan el trabajo autónomo. Esta es una de las funcionalidades que presenta Edpuzzle junto con la posibilidad de recortar los vídeos e incluir notas de voz o sustituir el audio original para adaptar los vídeos al público al que se dirigen.

Desde el punto de vista de la gestión Edpuzzle permite la creación de aulas virtuales donde los participantes se unen a través de un código facilitado por el profesor. También el profesor puede comprobar de manera inmediata qué alumnos vieron el vídeo, si los vieron completamente, si contestaron a las preguntas incluidas a lo largo de los vídeos y qué contestaron, lo que nos ayuda a comprender mejor cuáles son las dificultades principales que cada alumno ha encontrado en el trabajo.

- Lensoo Create: creación de vídeos. Convierte cualquier dispositivo Android en una pizarra digital con grabación de voz y escritura digital.

- Kahoot!: para la evaluación de los conocimientos adquiridos por el alumnado. Combina la dinámica de juegos con los beneficios de respuesta personal del alumnado, de manera que la clase se convierte temporalmente en un espectáculo de juego (Moya et al., 2016)

Se creó un aula virtual en Edpuzzle donde los alumnos encontrarían todos los vídeos que debían ver antes de las sesiones presenciales
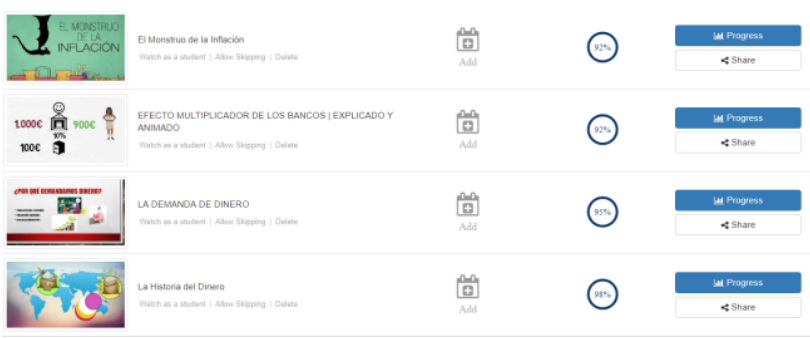

Figura 1. Página de inicio de Edpuzzle

Los vídeos utilizados se han obtenido de diferentes fuentes. Aquellos contenidos para los que se encontraron vídeos adecuados en YouTube han sido insertados directamente desde esta plataforma y editados con Edpuzzle. Para aquellos contenidos en los que no se han encontrado vídeos adecuados han sido creados con Lensoo Create y, posteriormente, incluidos en Edpuzzle.

Edpuzzle ha sido el entorno virtual en el que se ha sustentado el desarrollo de la experiencia. Al entrar en la plataforma los alumnos encontraban los diferentes vídeos que debían trabajar antes de las sesiones presenciales. Estos vídeos incluían puntos de participación para el alumnado, tales como preguntas abiertas o de opción múltiple como estrategia de motivación y gamificación, así como notas de voz y aclaraciones por parte del docente.

\subsection{Desarrollo de la unidad didáctica}

En la sesión inicial se hizo una introducción de la metodología a seguir en la unidad didáctica "El dinero y la política monetaria" exponiendo los cambios más importantes respecto a la metodología utilizada tradicionalmente en el aula. El cambio en el concepto de tareas para casa que se sustituirían por el trabajo de vídeos o textos, en el aula el profesor asumiría el papel de experto ayudándoles en las tareas que hacen normalmente fuera del aula. 
El desarrollo de la unidad didáctica estaba previsto para ocho sesiones que siguieron esta estructura:

- Resolución de dudas surgidas en el trabajo autónomo con los vídeos y textos recomendados a propuesta de los alumnos u observadas en las respuestas a las preguntas incrustadas en los vídeos.

- Breve debate sobre el contenido de los vídeos.

- Actividades propuestas para su realización en el tiempo de clase. Para la resolución en el aula de aquellas actividades que normalmente se han realizado de manera individual se han utilizado estructuras de trabajo cooperativo: a) "Think Pair Sharing" donde los estudiantes trabajan individualmente un tema que después discuten con una pareja para posteriormente compartirlo con toda la clase (Kagan, 1989); b) "1-2-4", donde primero cada alumno piensa cuál es la respuesta correcta a una pregunta; en segundo lugar se colocan en parejas intercambian sus respuestas y las comentan y, finalmente, todo el equipo debe decidir cuál es la respuesta más adecuada a la pregunta o preguntas que se les ha planteado.

- Durante la realización de las actividades el profesor resolverá las dudas que puedan surgir en el trabajo individual o grupal y que no han podido ser resueltas por los propios alumnos.

- Indicaciones sobre el material a trabajar en casa para la próxima sesión.

Para el desarrollo de la unidad didáctica se diseñaron las siguientes actividades para trabajar fuera del aula: 6 vídeos sobre los contenidos a tratar, visualizados de forma autónoma por los alumnos. 4 de ellos seleccionados de YouTube y 2 de elaboración propia, además de 3 textos.

\section{RESULTADOS Y DISCUSIÓN}

A continuación, se detallan los resultados obtenidos con los diferentes instrumentos de observación utilizados.

\subsection{Resultados de la guía de observación}

Tabla 1. Resultados de la observación participante

\begin{tabular}{lll}
\hline & Sí & No \\
\hline $\begin{array}{l}\text { El alumnado está contento al inicio } \\
\text { de la sesión }\end{array}$ & 90 & 10 \\
\hline $\begin{array}{l}\text { El alumnado participa en el debate } \\
\text { creado en torno al material trabajado } \\
\text { fuera del aula }\end{array}$ & & \\
\hline $\begin{array}{l}\text { El alumnado ha preguntado dudas } \\
\text { acerca de los contenidos del } \\
\text { material trabajado fuera del aula }\end{array}$ & & \\
\end{tabular}

\begin{tabular}{llll}
\hline El alumnado se ayuda y colabora & 80 & 20
\end{tabular} entre ellos en las actividades grupales

\begin{tabular}{l}
\hline $\begin{array}{l}\text { El alumnado requiere la ayuda del } \\
\text { docente }\end{array}$ \\
\hline $\begin{array}{l}\text { El tiempo programado es suficiente } \\
\text { para la terminar las tareas }\end{array}$ \\
propuestas
\end{tabular}

El alumnado muestra interés por $100 \quad 0$ comenzar con las actividades

Han surgido problemas técnicos que $15 \quad 85$ han ralentizado el transcurso de la sesión

\begin{tabular}{llll}
\hline El alumnado muestra iniciativa & 100 & 0 \\
\hline La dinámica del aula es participativa & 100 & 0 \\
\hline $\begin{array}{l}\text { Mantienen un ritmo de trabajo } \\
\text { autónomo }\end{array}$ & 00 \\
\hline $\begin{array}{l}\text { El alumnado participa en el debate } \\
\text { de cierre de la sesión }\end{array}$ & 20 \\
$\begin{array}{l}\text { El alumnado ha entendido las } 80 \\
\text { instrucciones dadas en la sesión } \\
\text { anterior sobre los materiales a } \\
\text { trabajar fuera del aula }\end{array}$ & 20 \\
\hline
\end{tabular}

\subsection{Resultados del cuestionario final de los alumnos}

En este apartado se exponen los datos más relevantes acerca de las opiniones del alumnado recogidas en el cuestionario final. En las siguientes gráficas se pueden ver valorados diferentes ítems en una escala Likert de 1 a 5 , siendo 1 totalmente en desacuerdo y 5 totalmente de acuerdo.

En el siguiente gráfico pueden observarse los resultados para los siguientes ítems del cuestionario:

- 15.- Encuentro la nueva forma de dar clase más entretenida. 
- 16.- Me gustaría que en las clases de Economía de Segundo de Bachillerato se siguiera el Nuevo modelo

- 19.- Recomendaría la nueva forma de dar clase a un amigo/a.

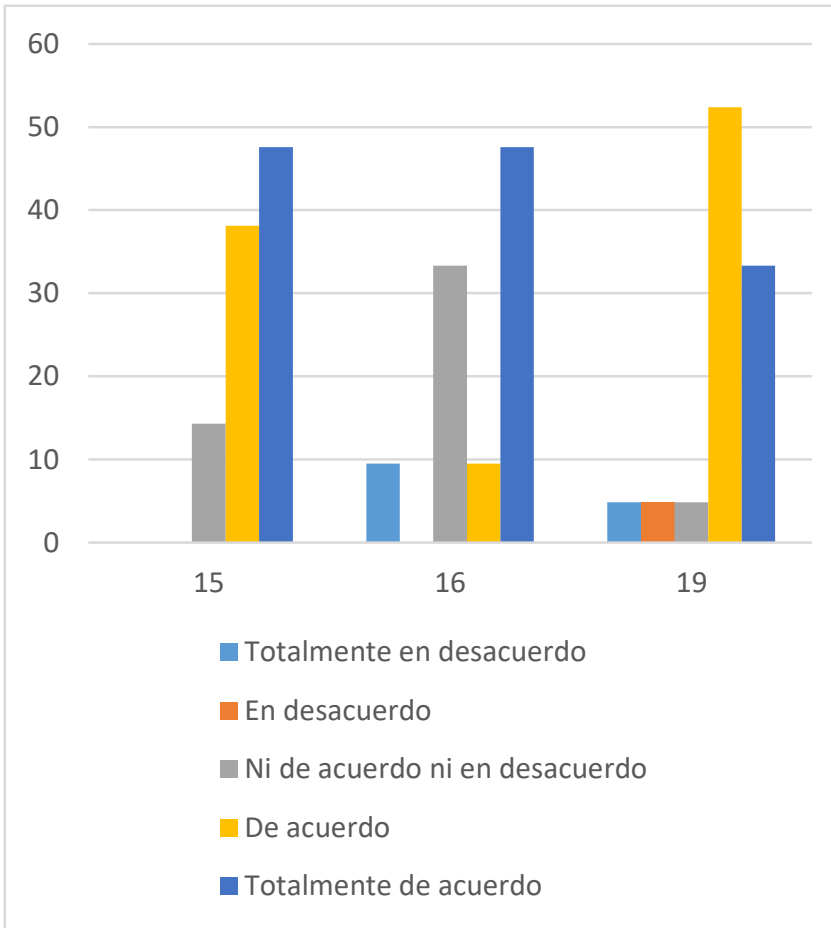

Figura 2. Respuestas a los ítems 15,16 y 19 sobre la "nueva forma de dar clase"

Como se puede observar en el gráfico anterior, teniendo en cuenta los alumnos que se encuentran de acuerdo y totalmente de acuerdo, el $85 \%$ de los participantes recomendaría el aula invertida a un amigo/a, solo el $10 \%$ no lo recomendaría y el $5 \%$ restante se manifiesta indiferente con esta afirmación. Estos resultados corroboran las conclusiones de diferentes autores sobre la satisfacción de los alumnos con la metodología manifestada a la hora de recomendarla a un amigo o amiga (Blázquez Ferrer et al., 2016; Calvillo, 2014; Rodríguez, Fernández y Vega, 2015).

Sin embargo, la respuesta, aunque positiva, no es tan contundente respecto a "Me gustaría que en las clases de Economía de segundo de Bachillerato se siguiera utilizando esta metodología, donde solo el $58 \%$ se muestra de acuerdo o totalmente de acuerdo con un porcentaje significativo de participantes, el $33,3 \%$, que se muestra indiferente y $9,5 \%$ en desacuerdo o totalmente en desacuerdo. De las experiencias anteriores consultadas no se extraen datos concluyentes en este sentido, las respuestas a esta pregunta difieren de los resultados obtenidos por Sáez y Ros (2014) que concluyen que, aunque la percepción que tienen los estudiantes sobre el uso de esta metodología es que es útil e interesante, la mayor parte no volvería a repetir esta experiencia. Otros autores (Bishop y Verleger, 2013; Jordán, Pérez y Sanabria, 2014; Rodríguez Learte et al., 2015) indican que, a pesar de la valoración positiva que los alumnos hacen del aula invertida, no confirman que prefieren esta metodología a la tradicional, si bien consideran la experiencia atractiva en general. No obstante, los resultados obtenidos en esta experiencia se acercan más a los de Roach (2014), donde un 76\% de los participantes se mostraban de acuerdo a seguir trabajando con la metodología del aula invertida.

En la Figura 3 se muestran los resultados de los ítems:

- 5.- Aprendo con el trabajo que hago en casa.

- 9.- Después de ver los vídeos voy con más confianza a clase.

- 12.- Me gusta ver los vídeos en casa.

- 34.- Prefiero las clases grabadas por el profesor a las explicaciones presenciales en la pizarra de clase.

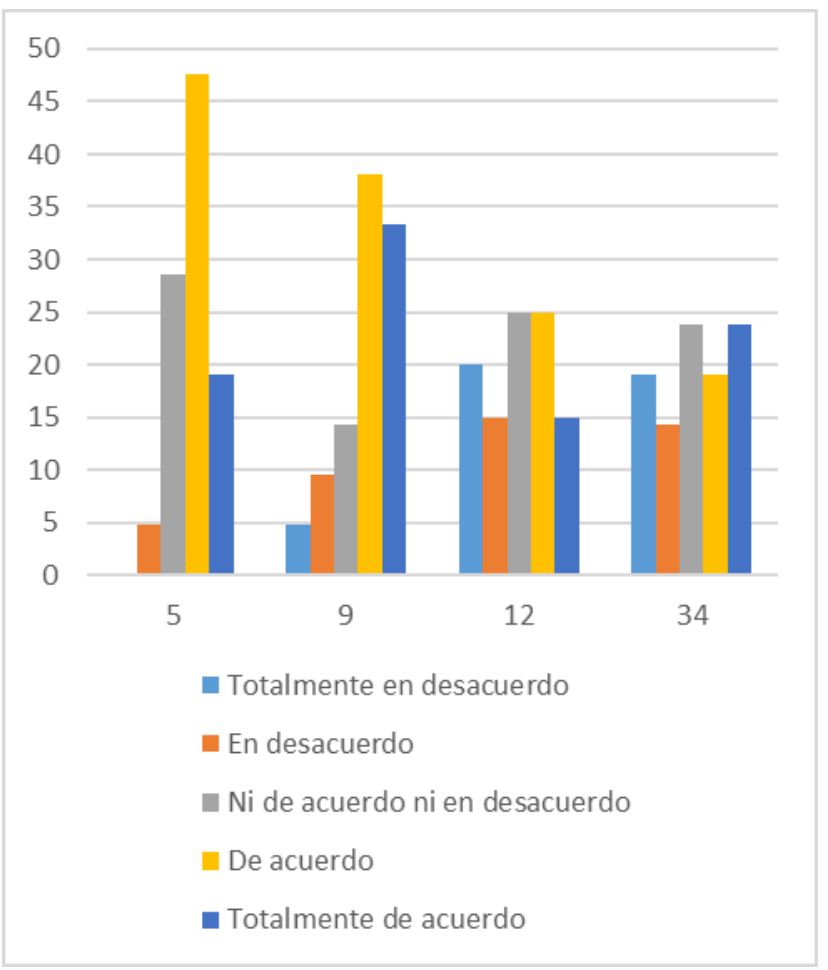

Figura 3. Respuestas a los ítems 5, 9, 12 y 34 sobre la actividad en casa y en clase

Respecto a la afirmación "me gusta ver los vídeos en casa", un $43 \%$ se encuentra de acuerdo o totalmente de acuerdo con esta afirmación, un $24 \%$ indiferente y un $33 \%$ en desacuerdo o totalmente en desacuerdo. Estos resultados se entienden mejor aportando los datos de la discusión mantenida; los alumnos consideran excesiva la cantidad de tareas que deben realizar en casa para el conjunto de 
materias y no reciben con agrado tareas bajo ningún formato. También inciden en el resultado de esta pregunta algunas incidencias con el uso de la plataforma Edpuzzle a las que se ha hecho referencia anteriormente.

En cambio, sobre la confianza con la que se asiste a clase después del trabajo previo en casa un $14,3 \%$ opina que no tiene más confianza a la hora de asistir a clase, un porcentaje similar, 14,3\%, indiferente y un $71,4 \%$ afirma que asiste con más confianza al aula después del trabajo realizado en casa, confirmando lo expuesto por Núñez y Gutiérrez (2016), Barreras (2016), Calvillo (2014) y Parra y Gutiérrez (2017).

Sobre su preferencia acerca de las clases grabadas o bajo el formato tradicional puede observarse que para el conjunto de la clase no existe una preferencia contundente, un $25 \%$ se muestra indiferente mientras que el $40 \%$ está de acuerdo o totalmente de acuerdo con la afirmación "prefiero las clases grabadas por el profesor que las explicaciones presenciales en la pizarra", mientras que un $35 \%$ está en desacuerdo o totalmente en desacuerdo. Algunos comentarios de los alumnos al respecto aclaran el porqué de una opción u otra: "las clases grabadas están muy bien pero al no estar el profesor presente no te puede resolver una duda al instante como podría hacerlo en clase", "en las clases con el profesor puedes preguntar la duda en el momento de la explicación" o "las clases grabadas están mejor porque puedes verlas todas las veces que quieras" coincidiendo con las limitaciones del modelo de aula invertida expuestas en el marco teórico (Nielsen, 2012; Wright, 2012).

En la figura 4 se muestran los resultados de los ítems:

- 23.- Las preguntas durante los vídeos me ayudan a entender los conceptos principales

- 25.- Durante los vídeos he tomado notas o apuntes

En dicha figura se observa que solo un $28,5 \%$ de los participantes afirma haber tomado notas $\mathrm{o}$ apuntes durante el visionado de los vídeos propuestos, este dato podría indicar que los participantes se han limitado simplemente a ver los vídeos. Como se expuso en el apartado dedicado a las herramientas utilizadas en el diseño de esta experiencia una de las funcionalidades de Edpuzzle es que permite incluir preguntas durante los vídeos. Un $71,4 \%$ se muestra de acuerdo o totalmente de acuerdo con la afirmación "las preguntas durante los vídeos me han ayudado a entender los conceptos principales".

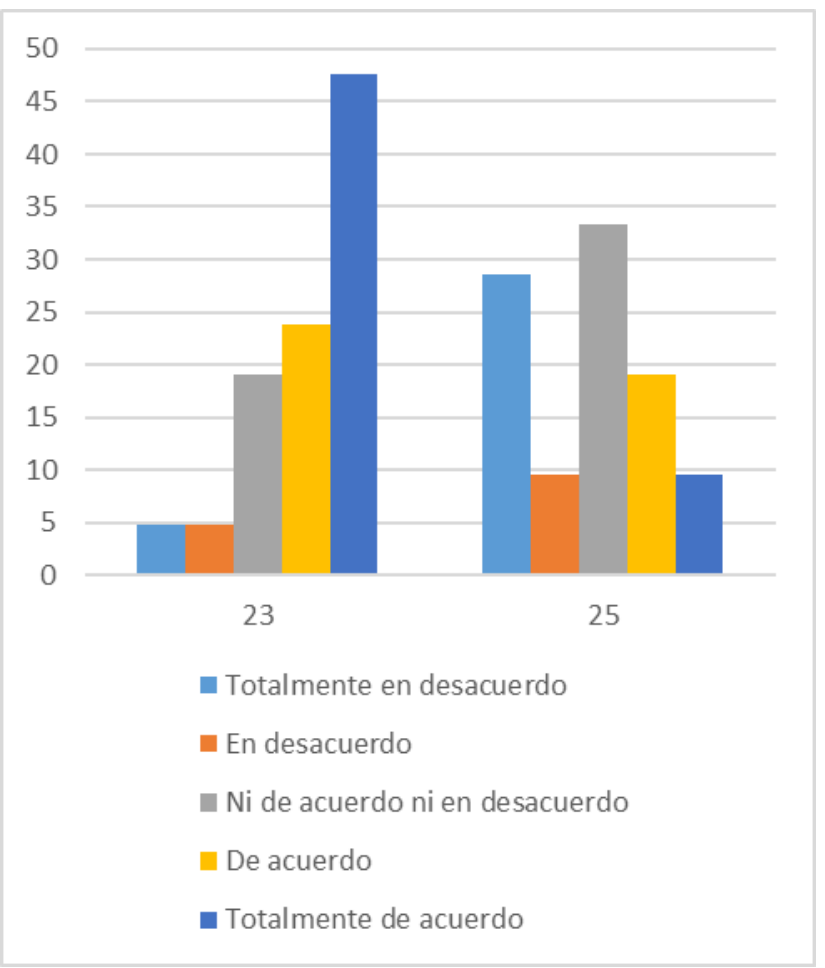

Figura 4. Respuestas a los ítems 23 y 25 sobre la actividad del alumno durante el visionado de los vídeos.

Relacionando ambas cuestiones los alumnos no han necesitado tomar notas o apuntes porque los conceptos principales se trabajaban ya con otros medios como las preguntas o aclaraciones insertadas en los vídeos constatando lo expuesto por Rodríguez Learte et al. (2015), "los alumnos prefieren visualizar vídeos sin tomar notas sobre el contenido de los mismos, el 80\% de los alumnos prefiere poner atención exclusivamente al vídeo" (p. 697).

Por último, se exponen los resultados de las preguntas referentes a la atención recibida por el profesor en clase y la ayuda recibida por sus compañeros de clase:

- 7.-El nuevo método hace que el profesor tenga más tiempo para resolver mis dudas y las de mis compañeros de forma individual.

- 8.-Con la nueva forma de trabajar mis compañeros/as me ayudan más en clase. 


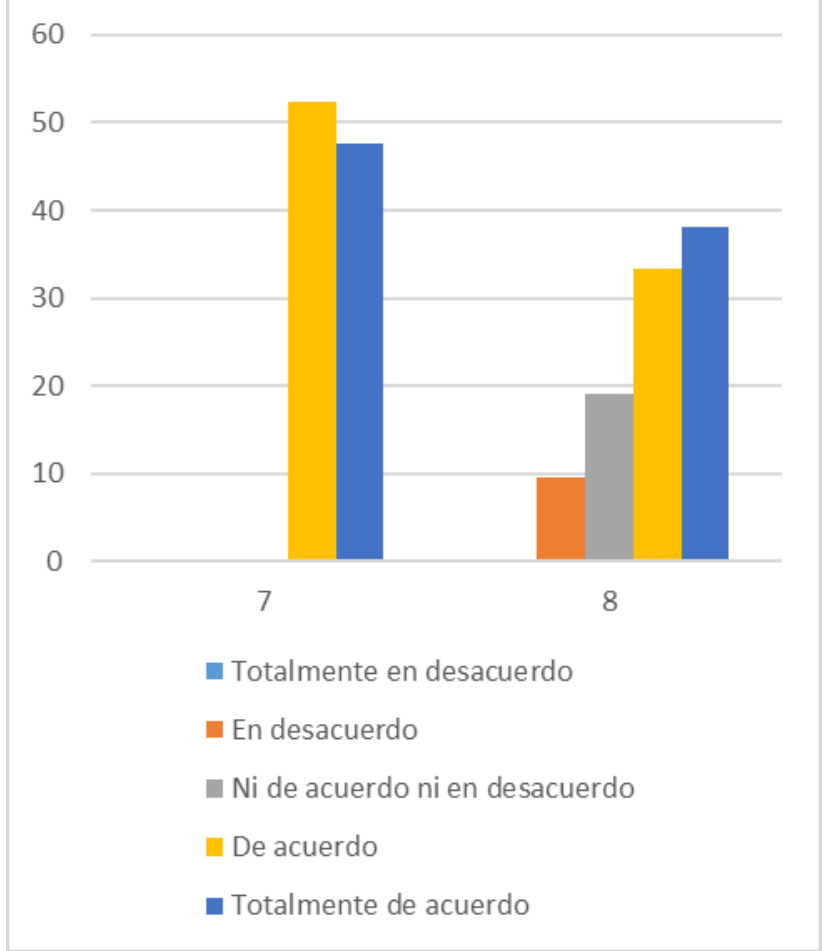

Figura 5. Respuestas a los ítems 7 y 8 sobre el tiempo de clase.

Como se puede observar en la figura 5 el $100 \%$ de los participantes afirman que con el aula invertida aumenta el tiempo que el docente puede dedicarles de manera individual. La ayuda prestada por los propios compañeros no arroja resultados tan contundentes: el $71,4 \%$ de los participantes se muestra de acuerdo con esta afirmación mientras el $9,5 \%$ está en desacuerdo y el $19 \%$ no observa ningún impacto de la metodología aplicada en la ayuda recibida por parte de sus compañeros. Estos datos coinciden con estudios anteriores en cuanto a la percepción del alumno del nuevo rol del profesor y facilidad para ayudar a los alumnos de manera personalizadas, y al aumento de ayuda entre los propios compañeros (Millard, 2012; Blázquez Ferrer et al., 2016; Calvillo, 2014; Fernández-Gámez y Guerra-Martín, 2016).

\section{CONCLUSIONES}

La implantación progresiva de las TIC en las aulas ha conducido a un cambio en el foco de las preocupaciones metodológicas del profesorado, que ha visto en ellas una poderosa herramienta con la que potenciar el aprendizaje del alumno y captar su atención. A menudo las TIC son usadas para cambiar métodos y enfoques educativos tradicionales (García-Barrera, 2013). A ello se añade que el aula invertida es un método en expansión (Bishop y Verleger, 2013; Hall y DuFrene, 2016;
Nguyen et al., 2016; Norman y Wills, 2015). De ahí nuestro interés en aplicar el método del aula invertida con un grupo de la asignatura de Economía de primero de Bachillerato evaluando la experiencia a través de la opinión de los estudiantes.

Tras finalizar la experiencia se puede concluir que la implementación del aula invertida ha sido muy satisfactoria. Los participantes han ido adaptándose paulatinamente a la nueva forma de trabajo y han aprendido a participar activamente en el aula, habiendo realizado sus tareas previas y las actividades de aula.

En general, el aula invertida es valorada positivamente por los participantes, el alumnado la encuentra más entretenida y afirman que la recomendarían a un amigo. Sin embargo, su valoración sobre la utilización en la misma asignatura el próximo curso es algo ambigua. Los participantes valoran positivamente el cambio en la dinámica del aula, aunque no se puede concluir que prefieran el formato de clases grabadas sobre las clases presenciales, pero sin duda agradecen la posibilidad de ver repetidas las lecciones todas las veces que necesiten y valoran la presencia del profesor en las clases presenciales para resolver dudas.

En cuanto a la atención recibida por parte del profesor $\mathrm{y}$ de sus propios compañeros, los participantes en general afirman que aumenta la ayuda prestada individualmente por parte del profesor y la del resto de sus compañeros de clase.

En el futuro y para confirmar las conclusiones de esta experiencia de innovación educativa, sería interesante ampliar el horizonte temporal de referencia, poder experimentar un curso completo o bien contar con distintos grupos de alumnos para poder comparar resultados.

\section{REFERENCIAS}

Barao Moreno, L., y Palau Martín, R. F. (2016). Análisis De La Implementación De Flipped Classroom En Las Asignaturas Instrumentales De $4^{\circ}$ Educación Secundaria Obligatoria Review of the Implementation of Flipped Classroom in the Core Subjects of 4 Year in Eso ( Obligatory Secondary Education ). Edutec. Revista Electrónica de Tecnología Educativa, (55), 1-13.

Barreras Gómez, M. A. (2016). Experiencia de la clase inversa en didáctica de las lenguas extranjeras a flippedclassroom experience in Didactics of Foreign Language. Educatio Siglo XXI, 34Asunción(1), 173-196. doi:10.6018/j/253281

Bergmann, J. y Sams, A. (2012) Flip your Classroom: Reach Every Student in Every Class Every day. International Society for Technology in Education

Bishop, J. L., y Verleger, M. A. (2013). The Flipped Classroom: A Survey of the Research. In Proccedings of 
the Annual Conference of the American Society for Engineering Education (p. 6219).

Blázquez Ferrer, M., Giner Pons, R., Jaime, I., Cortes Martínez, D., Ríos Cañavate, J., de Luís Margarit, A., Santamarina Siurana, M. (2016). Flipped classroom en el aprendizaje multidisciplinar colaborativo en diferentes Grados Universitarios. XIV Jornadas de Redes de Investigación en Docencia Universitaria. Investigación, innovación y enseñanza universitaria: enfoques pluridisciplinares. Alicante. Recuperado http://hdl.handle.net/10045/57093 (pp. 2414 - 2423)

Cabrera, F. (1987). Investigación evaluativa en educación. Madrid: Ed. Largo Caballero.

Cakir, C. (2016). TESOL plus TELOS: Teaching English as a Language of Open Sources (TELOS). In International Conference on Teaching and Learning English as an Additional Language GlobELT (pp. 600-603). Antalya. Recuperado de https://bit.ly/2JOHXNC

Calvillo Castro, A. J. (2014). El modelo Flipped Learning aplicado a la materia de música en el cuarto curso de Educación Secundaria Obligatoria: una investigaciónacción para la mejora de la práctica docente y del rendimiento académico del alumnado. Tesis doctoral. Universidad de Valladolid.

Carr, R., Palmer, S., y Hagel, P. (2015). Active learning: The importance of developing a comprehensive measure. Active Learning in Higher Education, 16(3), 173-186. doi:10.1177/1469787415589529

Fernández-Gámez D, G.-M. M. (2016). Aprendizaje inverso en formación profesional: opiniones de los estudiantes. International Journal of Technology and Educational Innovation, 2(1), 29-37. doi:10.20548/innoeduca.2016.v2i1.1048

Foldnes, N. (2016). The flipped classroom and cooperative learning: Evidence from a randomised experiment. Active Learning in Higher Education, 17(1), 39-49. doi:10.1177/1469787415616726

Fornons, V., y Palau, R. (2016). Flipped Classroom en la asignatura de matemáticas de $3^{\circ}$ de Educación Secundária Obligatória. Edutec. Revista Electrónica de Tecnología Educativa, 55, 1-17. doi:10.21556/edutec.2016.55.284

García-Barrera, A. (2013). El aula inversa: cambiando la respuesta a las necesidades de los estudiantes. Avances En Supervisión Educativa, O(19). Recuperado de https://avances.adide.org/index.php/ase/article/view/118/1 15

Gómez García, I., Castro Lemus, N., y Toledo Morales, P. (2015). Las flipped classroom a través del smartphone: efectos de su experimentación en educación física secundaria. Prisma Social. Revista de Ciencias Sociales, (15), 296-351. Recuperado de http://www.isdfundacion.org/publicaciones/revista/numer os/15/secciones/tematica/pdf/t_09_flippedclassroom_296-351.pdf

Hall, A. A., y DuFrene, D. D. (2016). Best Practices for Launching a Flipped Classroom. Business and Professional Communication Quarterly, 79(2), 234-242. doi:10.1177/2329490615606733

Harrison, C., Comber, C., Fisher, T., Haw, K., Lewin, C., y Lunzer, E. (2003). The impact of Information and Communication Technologies on Pupil Learning and Attainment. ImpaCT2. Recuperado de https://bit.ly/2JMrZU5

Jancsó, K. (2017). ¿Cómo darle la vuelta a la clase de ELE? El aula invertida y el uso de Edpuzzle y Powtoon en la enseñenza de español. Revista Electrónica Del Departamento de Estudios Hispánicos de La Universidad de Szeged, 1. Recuperado de www.hispanisztikaszeged.hu Jordán Lluch, C., Pérez Peñalver, M. J., y Sanabria Codesal, E. (2014). Experiencias Docentes Investigación del impacto en un aula de matemáticas al utilizar flip education. Pensamiento Matemático, 4(2). Recuperado de https://riunet.upv.es/bitstream/handle/10251/49189/Artícu lo flip Pensamiento Matemático.pdf?sequence $=2$ yisAllowed $=\mathrm{y}$

Kagan, S. (1989). The structural approach to cooperative learning. Association for Supervision and Curriculum development. Recuperado de http://www.ascd.org/ASCD/pdf/journals/ed_lead/el_1989 12_kagan.pdf

Llamazares Carballo, A. (2015). HU University of Applied Sciences Utrecht. El Guiniguada. Revista de Investigaciones $Y$ Experiencias En Ciencias de La Educación, 23(2014), 63-70.

Marqués, M. (2016). Qué hay detrás de la clase al revés (flipped classroom). Revista de Investigación En Docencia Universitaria de La Informática, 77-84.

Merla González, A. E., y Yáñez Encizo, C. G. (2016). El aula invertida como estrategia para la mejora del rendimiento académico. Revista Mexicana de Bachillerato a Distancia, 16(8), 67-77.

Millard, E. (2012). 5 Reasons Flipped Classrooms Work: Turning lectures into homework to boost student engagement and increase technology fueled creativity. University Business.com, 26-29. Recuperado de http://www.universitybusiness.com/article/5-reasonsflipped-classrooms-work..

Moya Fuentes, M. M., Carrasco Andrino, M. ., Jiménez Pascual, M. A., Ramón Martín, A., Soler García, C., y Vaello López, M. T. (2016). El aprendizaje basado en juegos: experiencias docentes en la aplicación de la plataforma virtual Kahoot. In XIV Jornades de Xarxes D'Investigació en Docència Universitària (pp. 1241-1254). Alicante: Universidad de Alicante. Recuperado de https://rua.ua.es/dspace/bitstream/10045/59136/1/XIVJornadas-Redes-ICE_090.pdf

Nguyen, B., Yu, X., Japutra, A., y Chen, C.-H. S. (2016). Reverse teaching: Exploring student perceptions of "flip teaching." Active Learning in Higher Education, 17(1), 51-61. doi:10.1177/1469787415616727

Norman, S., y Wills, D. (2015). Flipping your Classroom in Economics Instruction: it's not all or nothing, (May).

Núñez, A., y Gutiérrez, I. (2016). Flipped Learning Para El Aprendizaje Del Inglés. Edutec. Revista Electrónica de Tecnología Educativa, 56, 89-102.

Palazón-herrera, J. (2016). Vídeo interactivo como herramienta de apoyo al análisis musical en educación secundaria Interactive Video as a Tool of Support to the Musical Analysis in Secondary Education, 8, 412-428.

Parra Giménez, F. J., y Gutiérrez Porlán, I. (2017). Implementación y análisis de una experiencia de flipped classroom en Educación Musical. Innoeduca. International Journal of Technology and Educational Innovation, 3(1), 4-14.

Pérez Serrano, G. (2007) Modelos de Investigación Cualitativa en Educación Social y Animación Sociocultural. Madrid: Narcea.

Prieto Calvo, C., Santos Sánchez, M. J., Hernández Encinas, A., Moreno, M., Rodríguezpuebla, C., y Queiruga-Dios, A. (2016). Dispositivos Móviles como instrumentos para la Adquisición De Competencias en Materias de Ciencias. In VI Jornada de Innovación Docente de la Universidad de Valladolid (pp. 7-11). Valladolid. Recuperado de http://uvadoc.uva.es/bitstream/10324/18413/1/Dispositivo 
s-moviles-Jornada-Innovación-Docente.pdf

Prieto Espinosa, A., Prieto Campos, B., y Del Pino Prieto, B. (2016). Una experiencia de flipped classroom. In Actas de las XXII Jornadas sobre la Enseñanza Universitaria de la Informática (JENUI) (pp. 237-244). Almería.

Ranalli, J., y Moore, J. (2016). Targeted flipped classroom technique applied to a challenging topic. Proceedings Frontiers in Education Conference, FIE, 2016-Novem, 0-3. doi:10.1109/FIE.2016.7757603

Roach, T. (2014). Student perceptions toward flipped learning: New methods to increase interaction and active learning in economics. International Review of Economics Education, 17, 74-84. doi:10.1016/j.iree.2014.08.003

Robledo Ramón, P., Fidalgo Redondo, R., Arias Gundín, O., y Álvarez Fernández, L. (2015). Percepción de los estudiantes sobre el desarrollo de competencias a través de diferentes metodologías activas. Revista de Investigación Educativa, 33(2), 369. doi:10.6018/rie.33.2.201381

Rodríguez Domenech, M. Á., Nieto Moreno de Diezmas, E., y Sumozas García-Pardo, R. (2016). Las tecnologías en Educación. Hacia la calidad educativa. (Síntesis, Ed.) (1st ed.). Recuperado de http://10.0.49.251/pixelbit\%0Ahttp://search.ebscohost.co $\mathrm{m} /$ login.aspx direct=trueydb=ufhyAN=119485655ylang= esysite $=$ ehost-live

Rodríguez Learte, A. I., Fernández Vaquero, A., y Vega Avelaira, D. (2015). Desarrollo de metodologías de Flipped Classroom para asignaturas de ciencias básicas. In XI IJornadas Internacinoales de Innovación Universitaria. Educar para trasnformar (pp. 692-699).

Sáez López, J. M., Fernández Flores, M., y García González, J. L. (2012). Descubriendo Edmodo: beneficios del microblogging en educación de adultos . Campo Abierto, 31(1), 53-69. Recuperado de http://revistas.ojs.es/index.php/campoabierto/article/view/ 1196

Sáez Pizarro, B., y Ros Viñegla, M. P. (2014). UNA EXPERIENCIA DE FLIPPED CLASSROOM. In XI Jornadas Internacionales de Innovación Universitaria Educar para transformar. Recuperado de http://www.uem.es

Salas-Rueda, R.A., y Lugo-García, J.L. (2019). Impacto del aula invertida durante el proceso educativo superior sobre las derivadas considerando la ciencia de datos y el aprendizaje automático. EDMETIC, Revista de Educación $\begin{array}{llll}\text { Mediática } \quad y \quad \text { TIC, } & 8(1), & 147-170\end{array}$ doi:10.21071/edmetic.v8i1.9542

Salmerón, F.J. (2015) Aplicación de la metodología Flipped Classroom en Tecnologías de $1^{\circ}$ de ESO (Trabajo Fin de Máster). Universidad Internacional de la Rioja.

Sohrabi, B., y Iraj, H. (2016). Implementing flipped classroom using digital media: A comparison of two demographically different groups perceptions. doi:10.1016/j.chb.2016.02.056

Sosa Díaz, M.J. y Palau Martín, R.F. (2018) Flipped classroom para adquirir la competencia digital docente: una experiencia didáctica en la Educación Superior. Píxel-Bit. Revista de Medios y Educación, 52, 37-54 Recuperado de https://recyt.fecyt.es/index.php/pixel/article/view/62483. doi:http://dx.doi.org/10.12795/pixelbit.2018.i52.03.

Tourón, J., y Santiago, R. (2015). El modelo Flipped Learning y el desarrollo del talento en la escuela. Revista de Educacion, (368), 33-65. doi:10.4438/1988-592X-RE2015-368-288

Tucker, B. (2012). "The flipped classroom". Education Next, 12(1). (pp. 82-83).
Velilla Arrobas, T., Cazanave Sánchez, J., Cañizares Díaz, J. y Fernández Serrat, M. (2014). Herramientas didácticas para mejorar el rendimiento. Revista de Docencia Universitaria, 12(4), 397-413. Recuperado de redu.net/redu/index.php/REDU/article/view/.../pdf

Wright, S. (2012). Why I Gave Up Flipped Instruction. Recuperado June 19, 2017, de http://plpnetwork.com/2012/10/08/flip-love-affair/ 